\title{
Nonlinear rheology of glass-forming colloidal dispersions: transient stress-strain relations from anisotropic mode coupling theory and thermosensitive microgels
}

\author{
C M Amann ${ }^{1}$, M Siebenbürger $^{2}$, M Ballauff $^{2,3}$ and M Fuchs $^{1}$ \\ ${ }^{1}$ Fachbereich Physik, Universität Konstanz, 78457 Konstanz, Germany \\ ${ }^{2}$ Helmholtz-Zentrum für Materialien und Energie, 14109 Berlin, Germany \\ ${ }^{3}$ Institut für Physik, Humboldt-Universität zu Berlin, 12489 Berlin, Germany \\ E-mail: matthias.fuchs@uni-konstanz.de
}

\begin{abstract}
Transient stress-strain relations close to the colloidal glass transition are obtained within the integration through transients framework generalizing mode coupling theory to flow driven systems. Results from large-scale numerical calculations are quantitatively compared to experiments on thermosensitive microgels, which reveals that theory captures the magnitudes of stresses semi-quantitatively even in the nonlinear regime, but overestimates the characteristic strain where plastic events set in. The former conclusion can also be drawn from flow curves, while the latter conclusion is supported by a comparison to single particle motion measured by confocal microscopy. The qualitative picture, as previously obtained from simplifications of the theory in schematic models, is recovered by the quantitative solutions of the theory for Brownian hard spheres.
\end{abstract}

Keywords: colloidal dispersion, rheology, glass transition

\section{Introduction}

The nonlinear rheology of complex fluids exhibits a rich phenomenology which arises from the competition between external drive and internal dynamics [1]. The flow properties of dilute colloidal dispersions are well characterized by a Newtonian viscosity. Its value is dictated by Brownian motion and hydrodynamic interactions [2,3]. Dense dispersions of slightly polydisperse particles develop a viscoelastic response and approach a colloidal glass transition [4]. The resultant metastable soft solids can easily be melted by external (shear) flow and can be driven to stationary nonequilibrium states characterized by a nonlinear relation between stress and shear rate [5]. This so-called flow curve exhibits a Newtonian viscosity at small shear rates and for not too high densities, which is generally followed by a shear-thinning regime for intermediate shear rates. For parameters deep in the colloidal glass domain, a dynamic yield stress can be observed clearly which is also seen in computer simulations of glass forming systems at low temperatures [6]. Both phenomena (yielding and shear thinning) arise close to the colloidal glass transition from the decorrelation of the structural relaxation caused by the external flow; it can directly be seen in the intermediate scattering functions observed e.g. by confocal microscopy [7]. The colloidal rheology thus provides insights into the structural relaxation of glass forming systems, which bears similarities with plastic deformations in supercooled metallic melts [8]. Beyond the structural shear-thinning regime, various 
phenomena like a second Newtonian plateau, shear-thickening, or jamming are possible, with differences between glass-like and jamming-like response becoming clearer recently [9-11].

The quiescent colloidal glass transition is well described by mode coupling theory which focuses on the structural dynamics as captured in density correlation functions [12]. It was generalized to glass forming dispersions under homogeneous flows with arbitrary time-dependences in the integration through transients approach, albeit under the assumption that hydrodynamic interactions can be accounted for by a renormalization of short-time transport coefficients [13,14]. Schematic models were formulated which enable one to treat complicated flow histories in detail [15] and quantitative solutions of the microscopic theory for hard discs in two spatial dimensions were obtained and compared to computer simulations [16-18]. Only recently, the first quantitative flow curves and stress-strain relations were obtained considering Brownian hard spheres, including with a short ranged attraction, which could be compared to experimental data [19]. The rheological experiments were performed on solutions of thermosensitive core-shell microgels which allow a very fine control of the separation to the glass transition, so that broad windows in frequency and shear rate could be studied [5,11,20,21]. Moreover, their mechanical history can handily be erased using in situ temperature control, so that a detailed investigation of the transient stress-evolution and aging phenomena could be performed [18, 22, 23].

In this paper, the quantitative comparison of the nonlinear rheology as predicted by mode coupling theory with data measured on the thermosensitive microgels is continued. Stress-strain curves are considered which capture the transient stress evolution after switch-on of a constant shear rate. Extending the analysis of steady states in [19], the transient stress evolution is studied here. Also, more polydisperse samples are considered which, on the one hand, eliminates artefacts arising from crystallization in the experimental data, but, on the other hand, somewhat shifts the parameters compared to the less polydisperse samples. Therefore, an independent analysis of stationary flow curves precedes the analysis of the transient data. A comparison of theory with the shear-induced single particle motion as revealed by confocal microscopy [7] complements our discussion of the macroscopic rheology on the microscopic scale.

\section{Mode coupling theory in integration through transients framework}

We consider $N$ Brownian particles in a volume $V$ performing random walks relative to a flowing background. Its velocity field $\mathbf{v}=\dot{\gamma} y \hat{\mathbf{x}}$ is characterized by a homogeneous and constant shear rate $\dot{\gamma}$, which is switched-on at time $t=0$ [24]. The random walks are coupled by the forces among the particles. The effect of shear relative to Brownian motion is measured by the bare Péclet number $\mathrm{Pe}_{0}=\dot{\gamma} d^{2} / D_{0}$, which is built with the Stokes-Einstein-Sutherland diffusion coefficient $D_{0}$ of an isolated sphere with diameter $d$. An equation of motion for a normalized transient density correlator
$\Phi_{\mathbf{q}}(t)=\left\langle\delta \rho_{\mathbf{q}}^{*} \delta \rho_{\mathbf{q}(t)}(t)\right\rangle / N S_{q}$, with $S_{q}$ the equilibrium structure factor, is central to the integration through transients (ITT) approach, which obtains properties of the stationary state from following the transient evolution. This allows to evaluate averages in the initial equilibrium Gibbs-Boltzmann ensemble. The shear-advected wavevector $\mathbf{q}(t)=\left(q_{x}, q_{y}-\right.$ $\left.\dot{\gamma} t q_{x}, q_{z}\right)^{T}$ accounts for the affine particle motion with the flow. The correlator is affected by Taylor dispersion in an initial decay rate $\Gamma_{\mathbf{q}}(t)=D_{0} q^{2}(t) / S_{q(t)}$ and by structural dynamics described in a memory kernel:

$\dot{\Phi}_{\mathbf{q}}(t)+\Gamma_{\mathbf{q}}(t)\left\{\Phi_{\mathbf{q}}(t)+\int_{0}^{t} \mathrm{~d} t^{\prime} m_{\mathbf{q}}\left(t, t^{\prime}\right) \dot{\Phi}_{\mathbf{q}}\left(t^{\prime}\right)\right\}=0$.

The generalized (collective) friction kernel $m_{\mathbf{q}}\left(t, t^{\prime}\right)$ slows down because of the slow structural relaxation, which following mode coupling theory (MCT) [12] is approximated by a quadratic functional of the density correlators. The advection causes the kernel to measure fluctuations at increasing wavevector, $m_{\mathbf{q}}\left(t, t^{\prime}\right)=\bar{m}_{\mathbf{q}\left(t^{\prime}\right)}\left(t-t^{\prime}\right)$, with

$\bar{m}_{\mathbf{q}}(t)=\frac{1}{2 N} \sum_{\mathbf{k}+\mathbf{p}=\mathbf{q}}$
$\frac{S_{q(t)} S_{k} S_{p}}{q^{2}(t) q^{2}} V_{\mathbf{q k p}}(t) V_{\mathbf{q} \mathbf{k p}}(0) \Phi_{\mathbf{k}}(t) \Phi_{\mathbf{p}}(t)$,

where the vertex function is given using the Ornstein-Zernicke direct correlation function, $c_{q}=\frac{V}{N}\left(S_{q}-1\right) / S_{q}$, by

$$
V_{\mathbf{q k p}}(t)=\mathbf{q}(t) \cdot\left(\mathbf{k}(t) c_{k(t)}+\mathbf{p}(t) c_{p(t)}\right)(N / V) .
$$

The potential contribution of the shear stress $\sigma_{x y}$ (divided by volume) is approximated in a similar fashion as the friction kernel assuming that stress relaxations can be computed from integrating the transient density correlations

$\sigma_{x y}(t)=\frac{\dot{\gamma}}{2 V} \int_{0}^{t} \mathrm{~d} t^{\prime} \sum_{\mathbf{k}}\left[\frac{k_{x}^{2} k_{y} k_{y}\left(t^{\prime}\right)}{k\left(t^{\prime}\right) k} \frac{S_{k\left(t^{\prime}\right)}^{\prime} S_{k}^{\prime}}{S_{k\left(t^{\prime}\right)}^{2}}\right] \Phi_{\mathbf{k}}^{2}\left(t^{\prime}\right)$,

with $S_{k}^{\prime}=\partial S_{k} / \partial k$. Equation (4) is a MCT approximation to a generalized Green-Kubo relation [13]. Quantitative results follow from the equilibrium structure factor as only input. It captures the particle interactions and depends on the thermodynamic parameters like density and temperature.

To evaluate the MCT-ITT expressions for Brownian hard spheres, the Percus-Yevick approximation for the equilibrium structure is employed. It contains the packing fraction $\phi=$ $\frac{\pi}{6}(N / V) d^{3}$ as only thermodynamic parameter, which will be reported in relative separations, $\varepsilon=\left(\phi-\phi_{c}\right) / \phi_{c}$, from the (theoretical) glass transition packing fraction $\phi_{c} \approx 0.516$. Further information on the numerical MCT-ITT calculations, like the discretization of the wavevectors and time, can be found in [19], where also supplementary results on the transient stress evolution is contained.

\section{Thermosensitive core-shell microgels}

Aqueous core-shell microgels have been established as model system for the investigation of the colloidal glass transition under flow [5, 11, 18, 20-23]. The core consists of poly(styrene) (PS) onto which a thermosensitive crosslinked 


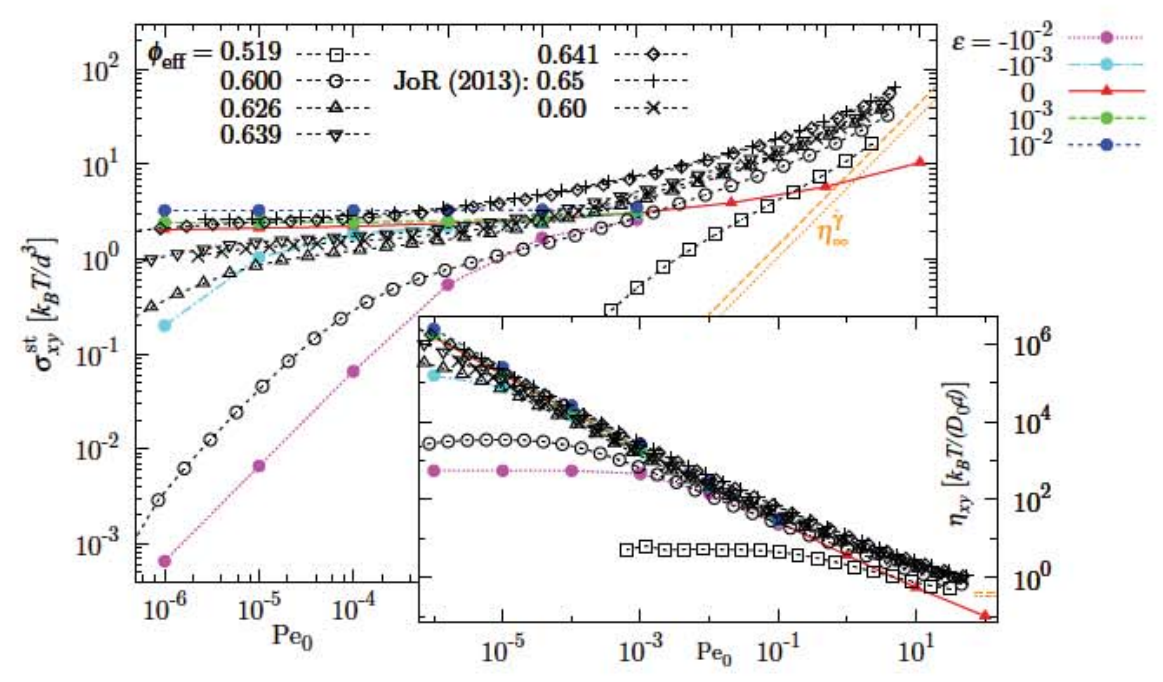

Figure 1. The main panel shows flow curves $\sigma_{x y}^{\text {st }}$ versus bare Péclet number $\mathrm{Pe}_{0}$ (colored symbols) numerically obtained from MCT-ITT; the relative packing fractions $\varepsilon$ are given in the legend and rescaling parameters in table 1 . Numerical values for six shear rates

$\mathrm{Pe}_{0}=10^{\{-6 ; \ldots ;-1\}}$ are connected by straight lines as guides to the eye (see text for additional data at $\varepsilon=0$ ). The open black symbols are experimental data obtained by [5]; they are plotted versus $\mathrm{Pe}_{\text {eff }}$ and effective packing fractions $\phi_{\text {eff }}$ are given in the legend. The high shear viscosity $\eta_{\infty}^{\dot{\gamma}}$ indicates the neglected hydrodynamic contribution. Standing and diagonal crosses are measured on other samples of the same PNiPAM system [18]. All experimental stresses are divided by $c_{y}=1.22$ and the density-dependent diffusion constant is estimated as $D_{s} / D_{0}=0.3$ [5]; it enters the experimental $\mathrm{Pe}_{\text {eff }}$. The inset shows the stationary viscosity $\eta_{x y}=\sigma_{x y}^{\text {st }} / \dot{\gamma}$.

network of poly( $\mathrm{N}$-isopropylacrylamide) (PNiPAM) is affixed. Below the lower critical solution temperature (LCST), the PNiPAM-shell is swollen and shows a linear shrinking up to $25^{\circ} \mathrm{C}$ with increasing temperature. Dispersions with a polydispersity of $9.3 \%$ crystallize and can be mapped onto the phase diagram of monodisperse hard spheres $[20,25,26]$. A polydispersity of $17 \%$, conveniently suppresses crystallization, but requires a mapping to determine the effective volume fraction $\phi_{\text {eff }}$ of the solution [5]. It could be established considering the short time dynamics which apparently depends on polydispersity only weakly. The glass transition lies around $\phi_{\mathrm{eff}}^{c}=0.64$ in this polydisperse system, which should be compared to $\phi^{c}=0.58$ found in the less polydisperse samples [20]. Whether the shift in critical packing fraction is solely due to polydispersity, or may arise from other sources like particle softness or the mapping itself, is unclear presently. The value $\phi_{\mathrm{eff}}^{c}=0.64$ should not be mistaken for random close packing which is shifted to higher packing fractions in polydisperse systems [27].

The rheological measurements were presented in $[5,18]$, where also the employed rheological protocol and additional details on the experimental system can be found. The bare Peclet numbers as introduced in section 2 follow from the viscosity $\eta_{s}$ of water and the hydrodynamic particle radius $R_{\mathrm{H}}=d / 2$ via $D_{0}=k_{\mathrm{B}} T /\left(3 \pi \eta_{s} d\right)$. Yet, as the theory neglects hydrodynamic interactions which are known to shift the short time transport coefficients, a mapping of the experimental and theoretical Peclet numbers is required. We take it from the density dependent short time self-diffusion coefficient $D_{s}$, which defines an effective Peclet number in the experimental system: $\mathrm{Pe}_{\mathrm{eff}}=\dot{\gamma} d^{2} / D_{s}$. The values of $D_{s}$ will be fitted by comparing experimental and theoretical data at corresponding Peclet numbers and will be reported in units of the experimental $D_{0}$ as determined from the solvent viscosity.
Our values of $D_{s}$ can then be compared to measurements in colloidal hard spheres reported in [28]. Hydrodynamic interactions also strongly affect the high shear viscosity $\eta_{\infty}^{\dot{\gamma}}$ which far exceeds the solvent one depending on particle surface properties like drainage [2]. Estimates for $\eta_{\infty}^{\dot{\gamma}}$ were extracted from the rheological measurement in the microgel dispersions by extrapolation, but will not be included into the present theoretical description. Rather, where appropriate, simple asymptotes using $\eta_{\infty}^{\dot{\gamma}}$ will delimit the range of applicability of the theory, which focuses on the rheology close to the glass transition which is dominated by structural relaxation processes.

\section{Results}

\subsection{Flow curves}

Flow curves give the stationary shear stress as function of the applied shear rate and thus describe the flow driven state in the most basic way. Because the experimental flow curves have been discussed in detail using schematic models of MCT-ITT $[5,18,20]$, the present discussion focuses on the quantitative description by microscopic MCT-ITT now available for Brownian hard spheres. The first point which needs to be discussed concerns the (neglected) hydrodynamic interactions induced by the solvent, which should not dominate over the structural contributions.

Figure 1 shows numerically obtained stress values for shear rates spanning five decades and for five packing fractions covering the close vicinity of the glass transition. The numerical costs for the full solution of the MCT-ITT equations (1)-(4) prevent us from obtaining a denser set of curves [19]. The relative separations in density start from a value $(\varepsilon=-0.01)$ where the Newtonian (low shear) 
viscosity has increased by slightly more than three orders in magnitude relative to the high shear viscosity $\eta_{\infty}^{\dot{\gamma}}$. The latter, which is dominated by hydrodynamics, is not contained in the theoretical description, but can be estimated from experimental data at Peclet numbers exceeding unity (Spanning the packing fractions shown in figure $1 \eta_{\infty}^{\dot{\gamma}}=1.82$ to 1.35 in units of $k_{\mathrm{B}} T /\left(D_{s} d\right)$ was measured by [5].). It is indicated in figure 1 by dashed lines. The increase of the stress relative to the high shear asymptote $\dot{\gamma} \eta_{\infty}^{\dot{\gamma}}$ is caused by structural dynamics and is the central concern of MCT-ITT. The corresponding increase of the shear-dependent viscosity $\eta_{x y}=\sigma_{x y}^{\text {st }} / \dot{\gamma}$ above the high shear limit is shown in the inset of figure 1 . As an aside, one may note that the representation of the data in flow curves is superior to the (nominally equivalent) viscosity plot. Flow curves provide a much more detailed view of the data as shifts among the curves and curvatures of the data sets become more noticeable. Comparing the numerical curves and the high shear asymptote would lead to a Peclet number around $\mathrm{Pe}_{0} \approx 10$ below which the structural stress dominates over the hydrodynamic one. Yet the experimental data indicate that there is a broad crossover-region extending down to $\mathrm{Pe}_{0} \approx 0.1$, where hydrodynamic and structural effects both determine the stress. Note that the existence of a second Newtonian viscosity plateau at $\eta_{\infty}^{\dot{\gamma}}$ depends on surface-drainage specifities of the particles [2,29] and for the present core-shell microgels has not been established conclusively [5]. In order to concentrate on the structural effects, the MCT-ITT calculations start at and extend below $\mathrm{Pe}_{0}=0.1$. The experimental data are taken to depend on the effective Peclet number Pe $\mathrm{e}_{\text {eff }}$ increased by the decrease of the short-time diffusivity $D_{s}$. The increase in $\mathrm{Pe}_{\text {eff }}$ indicates the smaller time scale separation of shearing and diffusion caused by slowing down of the short-time diffusion by hydrodynamic interactions. Just to give an estimate of the high shear predictions of MCT-ITT without inclusion of hydrodynamic interactions, the stress curve at the critical packing fraction is extended to higher shear rates; see the red line in figure 1 above $\mathrm{Pe}_{0}>0.1$. The requirement to accurately capture rapidly decaying dynamics at these high $\mathrm{Pe}_{0}$ prevents us from solving the complete MCT-ITT equations. Because the effect of the memory kernel becomes smaller at short times, we set $\bar{m}_{\mathbf{q}^{\prime}} \equiv 0$ in equation (2) for the calculation of $\sigma_{x y}^{\text {st }}\left(\mathrm{Pe}_{0}>0.1, \varepsilon=0\right)$. The numerical stress curve increases less strongly than would correspond to a second Newtonian viscosity plateau which is not predicted by theory in the region accessible in the present experimental system.

Concentrating on the flow curve below around $\mathrm{Pe}_{0}=0.1$, the structural contributions dominate in the flow curves as may be judged from the comparison of theory and experimental data. They can be made to match closely there. In order to reduce the numerical efforts, the separation parameters were not adjusted in the MCT-ITT calculations. The a priori chosen grid of Peclet numbers, $\mathrm{Pe}_{0}=10^{\{-6 ; \ldots ;-1\}}$, however, suffices to indicate that the numerically obtained increase of the shear stresses at small shear rates is compatible with the experimental observations. The quantitative comparison of the stress magnitudes provides an estimate of the error by MCT-ITT in calculating stationary stresses in nonequilibrium states. In figure 1, the experimental stresses were scaled down
Table 1. Rescaling parameters used to match the microscopic MCT calculations to the experimental stress-strain curves; see text for discussion.

\begin{tabular}{lll}
\hline$D_{s} / D_{0}$ & $c_{y}$ & $\gamma_{\text {res }}$ \\
\hline 0.3 & 1.22 & 3.4 \\
\hline
\end{tabular}

by $c_{y}=1.22$ indicating that theory slightly underestimates the steady stresses in the polydisperse dispersion. Scaling parameters from the present analysis are collected in table 1. Polydispersity and its unavoidable consequences on the effective packing fraction appears to be a factor that affects stress measurements and consequently the present tests of the theoretical stresses. While a detailed comparison of the linear response moduli from MCT and from less polydisperse PNiPAM microgels indicated that close to the equilibrium glass transition MCT underestimates the (Maxwell) shear modulus $G_{\infty}$ by a factor $c_{y}^{\mathrm{eq}}=G_{\infty}^{\mathrm{Ex}} / G_{\infty}^{\mathrm{mct}} \approx 1.4$ [20], the corresponding comparison for the polydisperse samples gave $c_{y}^{\text {eq }} \approx 2.3$ to 3.5 [5]. In relation to the equilibrium stress magnitudes, the steady state stresses therefore appear somewhat overestimated in the more polydisperse system. Our evaluation of the theory for a one-component system obviously can not address this dependence on polydispersity.

The main observations taken from the present section concern the regime where structural contributions dominate in the flow curves (below around $\mathrm{Pe}_{0} \leqslant 0.1$ ) and the verification of the stress magnitudes from MCT-ITT. More insights can be gained from the transient dynamics leading to the steady state.

\subsection{Stress-strain relations}

Transient stresses as function of accumulated strain $\gamma=\dot{\gamma} t$ after switch-on of a constant shear rate at time $t=0$ ('startup flow') reveal the complete evolution of the response of the dispersion to enforced flow. A dense dispersion, which forms a soft solid when left unperturbed, responds with an initial linear regime where the shear modulus $G_{\infty}$ of the solid can be determined from $\sigma_{x y}=G_{\infty} \gamma$. At long times or large strains, respectively, the stress approaches the flow-curve value $\sigma_{x y}^{\text {st }}$. Experiments [30-32] and simulations [18,33-35] show that strains of order unity suffice to reach the steady state. Between both regimes, a stress overshoot is the hallmark of the yielding of colloidal glasses. It can be seen for small shear rates $\left(\mathrm{Pe}_{0} \ll 1\right)$ which nevertheless exceed the inverse internal structural relaxation time so that the dressed Peclet or Weissenberg number $\mathrm{Pe}=\dot{\gamma} \tau$ is large, Pe $\gg 1$. The stress overshoot has been discussed in mesoscopic descriptions [36-39] where its connection to shear-banding and aging was explored.

Simulations and confocal microscopy in colloidal dispersions reveal that stress-overshoots can also arise in homogeneous flows starting from equilibrated initial states $[18,30-32,34,35]$. This case can be modeled by schematic models of MCT-ITT, which was discussed in detail in [18] including data from experiments, simulations and microscopic MCT-ITT in two dimensions. Yet, the stress-overshoot is a transient feature transcending the universal picture captured 
in schematic models. In MCT-ITT it arises from a negative contribution in the stress-vertex, viz. the wavevector dependent prefactors contained in the square bracket in equation (4). The subsequent time-integration gives the stress-overshoot with its maximum position at the vanishing of the (time-) integrand in equation (4). The magnitude and location of the stressovershoot thereby provide a close look at the accuracy of the microscopic MCT-ITT approximations and at the concept of wave-vector advection, viz. a time-dependent wavevector, entering equation (4).

The mechanism in MCT-ITT causing a stress-overshoot has been identified in [34] and its dependence on the shape of the pair-potential been explored $[19,40]$. Without the wavevector-advection, the stress-vertex in equation (4) is a complete square and thus positive, even though it contains the derivative of the static structure factor, which oscillates around zero. Wavevector-advection causes one of the k-dependent terms to change with time and thus dephases the complete square. The change of the transient stress, $\frac{\mathrm{d}}{\mathrm{d} t} \sigma_{x y}(t)$, which follows the decay of pairs of density fluctuations with wavevectors $\mathbf{k}$ and $-\mathbf{k}$ (whose correlators $\Phi_{\mathbf{k}}(t)$ agree), thus becomes negative when the oscillations in $S_{k(t)}^{\prime}$ are shifted relative to $S_{k}^{\prime}$ by around half a period. This behavior in MCT-ITT has been termed anelastic as it arises from the affine advection of a vertex irrespective of the decay of a density correlator which captures plastic relaxation [41]. As $S_{k}$ varies most rapidly around its peak at $(2 \pi$ times) the inverse of the average particle separation, it is this region of maximal $S_{k}^{\prime}$ which contributes most strongly in turning the stress-vertex negative. Local structural rearrangements are thus held responsible by MCT-ITT for the stress-overshoot in packing dominated glass forming systems. It arises in the anelastic regime where the glass structure yields and also is affected by plastic events, as the density correlators decay at comparable strains (see section 4.3 for a discussion of the latter). Because fully microscopic solutions of the MCT-ITT equations are now available, it is possible to test this anelastic mechanism and the accuracy of the stress-vertex in comparison to experimental data, which is the main aim of the present contribution.

Stress-strain relations at two different packing fractions are available for the test of microscopic MCT-ITT; one at $\phi=0.60$ below and one at $\phi=0.65$ above the estimated glass transition at $\phi_{c} \approx 0.64$ [18]. Equilibrated quiescent states can be prepared in the microgels systems for both packing fractions by pre-shearing at high $\mathrm{Pe}_{0}$ and then waiting for more than $10^{4} \mathrm{~s}$. This holds because even above the MCT glass transition, a slow relaxation process with roughly this relaxation time exists in the samples $[5,18,20]$. This ultra-slow relaxation process, which leads to finite longest relaxation times $\tau$ and thus to finite dressed Peclet numbers Pe, will be neglected in the following as Pe $\gg 1$ can be achieved, nevertheless. Note that the curves in figures 2 and 3 were aged for $600 \mathrm{~s}$ only which incurs some aging effects especially at small $\mathrm{Pe}_{0}$; see figure 6 in [18].

Figure 2 shows transient shear stresses $\sigma_{x y}$ in startup-flow as function of accumulated strain $\gamma=\dot{\gamma} t$ for different bare Péclet numbers $\mathrm{Pe}_{0} \leqslant 0.1$, where the analysis in section 4.2 indicated that structural effects dominate the stress. Data are shown which were numerically calculated with 3d MCTITT and measured in the polydisperse PNiPAM samples at comparable $\mathrm{Pe}_{0}$ [18]. All experimental curves of the microgels are divided by $c_{y}=1.22$, the scaling factor obtained in section 4.2. The comparison is made at corresponding Peclet numbers where the shift of the short-time diffusion coefficient $D_{s}$ relative to $D_{0}$ is taken into account; viz. experimental data are given for $\mathrm{Pe}_{\text {eff }}$ using $D_{s}$ from table 1 . The experimental sample is in a fluid state below the MCT glass transition and for the comparison numerical curves at relative separation $\varepsilon=-0.001$ are chosen. Note that this value of $\varepsilon$ was not optimized for a fit but selected from a small set of available numerical solutions. Figure 1 indicates that the theoretically predicted Newtonian viscosity (and thus $\tau$ ) is smaller than measured. We therefore concentrate on the limit of large Peclet numbers, where the precise value of $\tau$ does not matter (The stress-strain curves at the lowest $\mathrm{Pe}_{0}$ both in experiment and theory in figure 2 approach $\mathrm{Pe} \approx 1$ and thus change shape. The stress-overshoot becomes smaller and the stress almost monotonously crosses over from the initial linear regime to the steady state. This needs to hold when approaching the limit of a Newtonian fluid.). The most characteristic feature of the stress-strain curves is the stress-overshoot which heralds the yielding of the glass structure present for times short compared to $\tau$. In the experiments its position $\gamma_{*}$ varies around a value $\gamma_{*}=0.1$ in a systematic fashion with shear rate; shifting to larger values for higher shear rates [30,31]. Microscopic MCT-ITT recovers this trend, yet predicts values which are around a factor of 3.4 too large. Apparently theory requires a larger strain for the crossover from the anelastic to the plastic regime. Rescaling the theoretical stress-strain curves by $\gamma_{\text {res }}$, as is done in figures 2 and 3 , enables one to concentrate on their functional forms and on their dependences on control parameters. This reveals that the numerical data recover the trend of $\gamma_{*}$ to increase with shear rate. The stress-release after yielding in theory is somewhat smaller and smoother than observed experimentally. Theory also recovers the trend of the initial (an-) elastic regime to become stiffer with increasing $\mathrm{Pe}_{0}$, yet quantitatively the trend is overestimated. This stiffening arises from the merging of the shear-driven structural relaxation and the short-time relaxation, which is affected by hydrodynamic interactions. The temporal resolution provided by transient stress strain curves enables one to observe these (quantitative) differences even though the steady flow curves are still reasonably described in figure 1 at these $\mathrm{Pe}_{0}$.

Figure 3 shows stress-strain relations for a glassy state, viz. for an experimental state at $\phi=0.65$ whose flow curve approaches a dynamic yield stress for decreasing shear rate, at least within the experimental time window; see figure 1. According to MCT-ITT the interpretation of the stress-strain curves is simpler in a yielding glassy state than in a fluid state. The crossover to the fluid-like response for small shear rates is absent and MCT-ITT predicts stress-strain curves to approach a scaling function for $\mathrm{Pe}_{0} \rightarrow 0$. This is the analogy to the yield stress in the flow curves and observable in the numerical data for the two lowest $\mathrm{Pe}_{0}$ in figure 3. The experimental data do not show this trend consistently. The experimental flow curve 


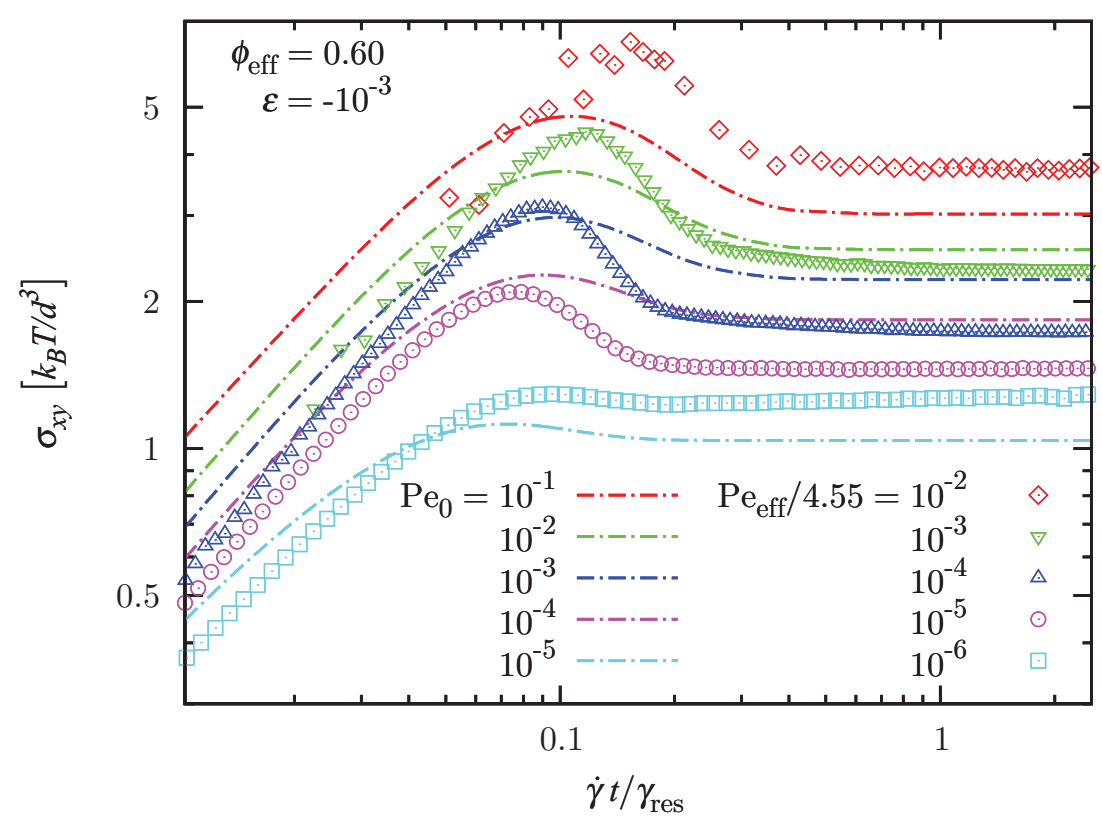

Figure 2. Transient shear stress $\sigma_{x y}$ as a function of accumulated strain $\dot{\gamma} t$ for a fluid state. Curves at different bare Péclet numbers Pe 0 calculated with $3 \mathrm{~d}$ MCT are compared with PNiPAM experiments from [18]. The separation to the transition is $\varepsilon=-10^{-3}$ in the MCT-ITT calculation, while the experimental packing fraction is $\phi_{\text {eff }}=0.60$. MCT-ITT curves are rescaled by $\gamma_{\text {res }}=3.4$ and experimental curves divided by $c_{y}=1.22$; see table 1 .

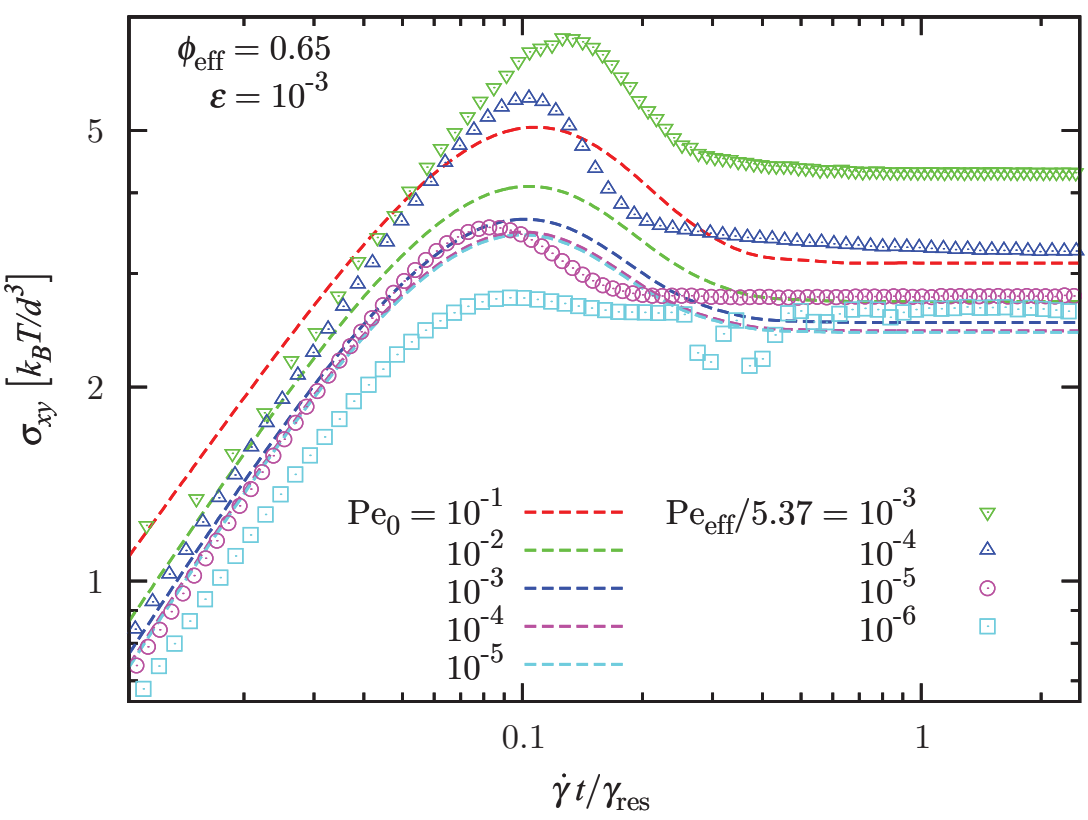

Figure 3. Transient shear stress $\sigma_{x y}$ as function of accumulated strain $\dot{\gamma} t$ for a glassy state. Curves at different bare Péclet numbers Pe 0 calculated with 3d MCT are compared with PNiPAM experiments from [18]. The separation to the transition is $\varepsilon=+10^{-3}$ in the MCT-ITT calculation, while the experimental packing fraction is $\phi_{\mathrm{eff}}=0.65$. MCT-ITT curves are rescaled by $\gamma_{\text {res }}=3.4$ and experimental curves divided by $c_{y}=1.22$; see table 1 .

is well compatible with a dynamical yield stress in figure 1 , while the experimental stress-strain curves do not collapse onto a master-curve for the two lowest $\mathrm{Pe}_{0}$ in figure 3. The difference possibly arises from a too short equilibration time before the start of the measurement affecting the low shear rate data; for a discussion of this effect at the larger of the two $\mathrm{Pe}_{0}$ see figure 6 in [18]. As we are unable to resolve this issue for the lowest shear rate curve, we neglect it for the following discussion.
Considering the curves at the three higher shear rates, MCT-ITT captures all observed trends qualitatively with quantitative differences already present in the fluid state. Considering the shape of the master-function valid in the limit of vanishing $\mathrm{Pe}_{0}$, theory and experiment at $\mathrm{Pe}_{\text {eff }}=$ $5.3 \times 10^{-5}$ closely resemble each other. The characteristic strain $\gamma_{*}$ of the maximum is overestimated by MCT-ITT by the same factor as below the glass transition, so that the same rescaling factor $\gamma_{\text {res }}$ from table 1 can be chosen to match 


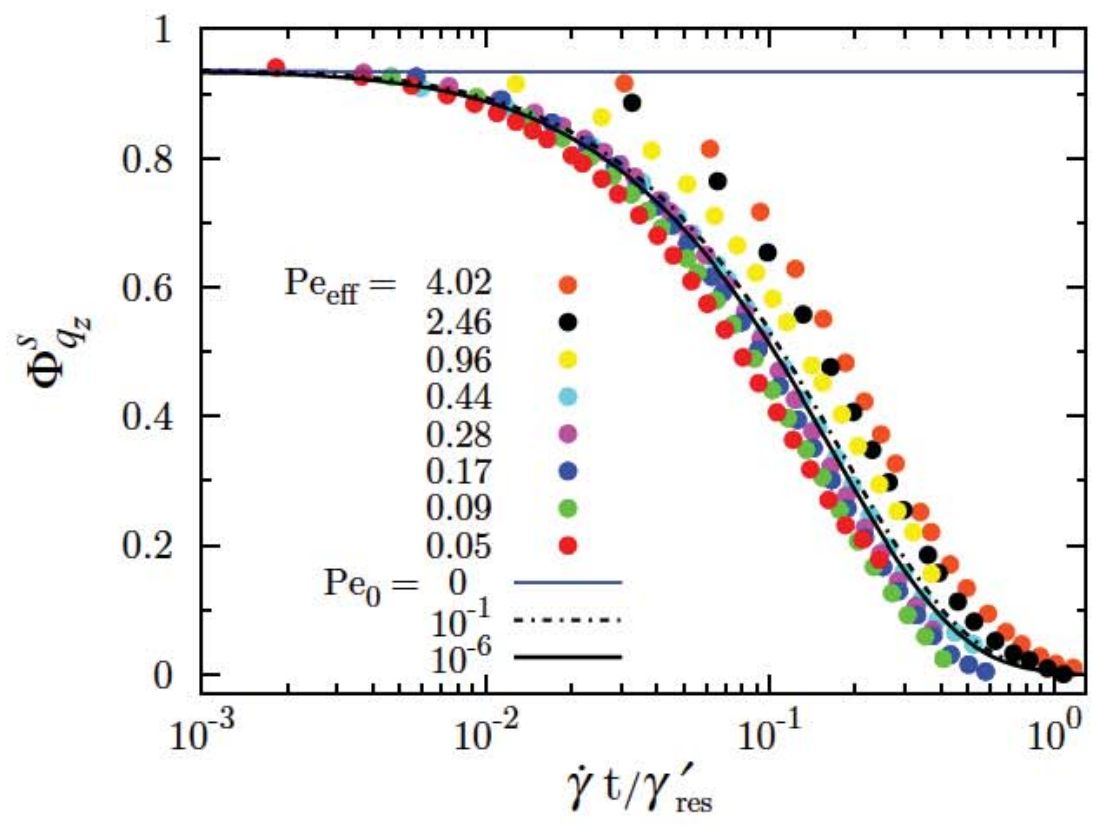

Figure 4. Symbols: steady-state incoherent density correlators $\Phi_{q_{z}}^{\mathrm{Ex}}(t)$ of a PMMA dispersion [7] as functions of strain, $\gamma=\dot{\gamma} t$, for several effective bare Péclet numbers $\mathrm{Pe}_{\mathrm{eff}}$ (see legend) at wavevector $q \stackrel{q_{z}}{=} 7.6 / d$ in vorticity direction $\left(\mathrm{q}=q e_{z}\right)$. The relative packing fraction is $\varepsilon_{\mathrm{Ex}} \simeq 0.07$. For comparison, coherent transient $3 \mathrm{~d}-\mathrm{MCT}$ correlators $\Phi_{\mathrm{q}}(t)$ are plotted as black lines versus rescaled strain $\dot{\gamma} t / \gamma_{\mathrm{res}}^{\prime}$ with $\gamma_{\text {res }}^{\prime}=33$; see legend for $\operatorname{Pe}_{0}$. Their parameters are $q=7 / d$ and $\phi=0.5257(\varepsilon \simeq 0.02)$. The $\Phi_{\mathrm{q}}(t)$ have been linearly interpolated from $\varepsilon=2 \cdot 10^{-2}$ and $\varepsilon=1 \cdot 10^{-2}$. The quiescent correlator $\left(\mathrm{Pe}_{0}=0\right)$ is shifted to agree with the correlator of $\mathrm{Pe}_{0}=10^{-1}$ at short times.

theory and experiment. Increasing $\mathrm{Pe}_{0}$, the stress-overshoot increases, moves to slightly larger strains and is followed by a stronger stress-release; all effects are present both in theory and experiment, but are stronger in the PNiPAM data.

The transient stress evolution in startup flow directly tests equation (4) where a stress-overshoot can only arise from negative contributions of the stress-vertex (the contents of the square bracket equation (4)) weighted by the spatially resolved density correlation functions which encode plastic structural rearrangements. The quantitative comparison with the microgel data points to an overestimate of the strain required for yielding, whose precise origin can be further investigated by considering density correlation functions directly. This will be done in the next section.

\subsection{Stationary time-dependent density fluctuations}

The central functions encoding structural rearrangements in MCT-ITT are the transient collective density correlators $\Phi_{\mathbf{k}}(t)$ obeying equations (1) to (3). While they have not been measured for the PNiPAM system, stationary incoherent density correlation functions have been measured by confocal microscopy in a colloidal poly (methyl methacrylate) (PMMA) dispersion, which is considered a hard sphere model system [7]. These measurements by Besseling et al clearly exhibit the speeding-up of the structural relaxation under shear in a state that is above the MCT glass transition without flow. This effect is central to MCT-ITT and contained in schematic and fully microscopic models. An analysis of the PMMA scattering data using an isotropically averaged hard sphere model (ISHSM) already indicated that MCT-ITT contains the shear-induced decay but requires larger strains for decay of density correlations than the confocal data. A matching to the data required $\gamma_{\text {res }}^{\text {ISHSM }}=30$ [24], far larger than the rescaling of the stress-strain curves found above in section 4.2.

Figure 4 shows a comparison of the numerically obtained transient collective correlator $\Phi_{\mathbf{q}}(t)$ with the steady-state incoherent density correlator $\Phi_{\mathrm{q}}^{\mathrm{Ex}}(t)$ of the PMMA dispersion, which was measured by confocal microscopy. In both cases, the magnitude of the wavevector corresponds to the peak of the quiescent structure factor $S_{q}$ and points in the neutral/ vorticity direction taken to be along $z$-axis. The packing fraction $\phi^{\mathrm{Ex}}=0.62$ of the experiment corresponds to a glass with about $\varepsilon_{\mathrm{Ex}} \simeq 0.07$; the data is taken from [7]. The given effective bare Péclet numbers $\mathrm{Pe}_{\mathrm{eff}}=\dot{\gamma} d^{2} / D_{s}$ of the experiment were estimated with a short-time self-diffusion coefficient $D_{s} \simeq D_{0} / 10.1$, with $D_{0}$ the dilute diffusion coefficient; the value (different from the PNiPAM one) is chosen based on measurements in [28] and may depend on packing fraction, polydispersity and hydrodynamic solvent flows close to the particle surface. The assumption to compare a transient and a stationary correlator has been addressed using Brownian dynamics simulations in [42]. The assumption to compare a coherent and an incoherent correlator at the peak in $S_{q}$ is based on [17], where comparable relaxation times were found in the MCT-ITT calculation in $d=2$. The present comparison, which is dictated by the lack of stationary incoherent correlators from theory, thus is rather crude but, we expect, is sufficient to identify a quantitative difference between theory and experiment. The matching in figure 4 requires to rescale the theoretical strain scale by $\gamma_{\text {res }}^{\prime}=33$, indicating that fully-anisotropic MCT-ITT predicts correlators to relax at strains which are appreciably too large compared to the experimental data (Note that the experimental data are 
plotted versus $\gamma=\dot{\gamma} t$ directly in figure 4.). Besides this quite large deviation in the strain scale, the qualitative agreement between the theoretical master-curve and data at small Peclet numbers is quite good. This supports the connection between the local structural relaxation and the macroscopic rheology encoded in MCT-ITT, which is the basis for its simplifications in schematic models. A similar comparison using the semischematic ISHSM can be found in [24], there with $\gamma_{\text {res }}^{\prime}=30$. The full $3 \mathrm{~d}$ calculation is thus quite similar in its $\gamma_{\text {res }}^{\prime}$ and decays even a bit slower in vorticity direction. This appears reasonable, because the ISHSM averages (heuristically) over all (faster relaxing) orientations of $\mathbf{q}$.

The comparison to the experiment reveals two differences. The first concerns the very different strain value which characterizes the decay of the experimental as compared to the theoretical density correlators. While this difference is affected by the simplification to compare transient and stationary correlators, the deviation is too big to arise from this aspect alone [42]. It is noteworthy that the theoretical stress-strain relations at very comparable parameters are characterized by a strain value that is far better in agreement with experiment than the one in the density correlators. This only holds in the fully anisotropic MCT-ITT calculation-note that stress and density fluctuations relax at comparable strains in the ISHSM [43] - and supports the quantitative accuracy of the stress-vertex in equation (4). Apparently it is the plastic decay encoded in the transient density correlators which sets in at too large strains only, while the wavevector advection in the stress-vertex acts on the proper strain-scale. The second difference concerns the lack of a yielding master curve in the experiment [7]. The experimental data rather exhibits a drift of the final decay to smaller strains with decreasing $\mathrm{Pe}_{\text {eff }}$. Besseling et al attributed it to a power-law $\tau_{\mathbf{q}} \propto \dot{\gamma}^{-0.8}$ which would be different from the asymptotic prediction of MCT-ITT $\tau_{\mathbf{q}} \propto \dot{\gamma}^{-1}$. A possible explanation for this could be that the approach to the master-function for vanishing shear rate takes place at appreciably different $\mathrm{Pe}_{0}$ in the experiment than in the theory. The actual range of experimental $\mathrm{Pe}_{\mathrm{eff}} \mathrm{starts}$ at the upper limit and far exceeds the range of the $\mathrm{Pe}_{0}$ in the numerical calculation. Yet, figure 4 shows that the deviations from scaling are larger than expected from theory, which is reminiscent of the broad crossover-region between structural and hydrodynamic stresses in figure 1. Presently anisotropic MCT-ITT can not address these crossovers quantitatively.

\section{Conclusions}

We have studied the quantitative accuracy of the microscopic mode coupling theory for the transient stress evolution of glass forming microgel dispersions in constant shear flow extending large-scale numerical calculations from [19]. Supporting earlier work using simplifications of the theory in schematic models, the qualitative phenomena in the stress-strain relations were recovered. Stress magnitudes are predicted with quantifiable errors which are comparable to the uncertainties in the experimental system stemming from e.g. polydispersity. We consider this as verification that MCT-ITT captures the nonlinear structural rheology of glass forming systems around the colloidal glass transition. This supports the use of schematic models within MCT-ITT, where spatial correlations are neglected and the derivation of constitutive equations from them, like the upper-convected Maxwell model with shear thinning [43] as used first by White and Metzner [44]. The latter approach to constitutive equations via the intermediate step of schematic models was recently generalized to tensorial and time-dependent flows [15] and applied to channel flow [45].

Quantitative agreement appears far better in $d=3$ than in $d=2$ where comparison to Brownian dynamics simulations indicated [17] that a larger rescaling of the strain scale is required than in the present analysis of stress-strain relations. In $d=2$ this error also affected the stress magnitudes causing MCT-ITT to overestimate the steady stress at the glass transition by an order in magnitude [16]. The present calculation indicates that the stress-vertex fares far better in $d=3$ and reduces the error to the characteristic strain of yielding to a factor around three. Steady stresses are predicted even more accurately. Interestingly, keeping the full anisotropic structure of the memory-kernels of MCT-ITT improves the description of the stress-strain relations but not of the transient density correlators compared to an isotropically averaged semi-schematic model [24]. This may point to the importance of an additional memory kernel $\Delta(\mathbf{q}, t)$ [24], which had been identified in the MCT-ITT equations of motion for the transient correlators by Chong and Kim [46] and by Suzuki and Hayakawa in a more complicated system [47], but has not been evaluated yet.

The presented large-scale numerical calculations now open the way to also describe the distorted structure under shear (work in progress), which will provide further insights into the structural mechanisms by which a colloidal glass yields under shear.

\section{Acknowledgments}

We thank F Frahsa for help with the analysis and the Deutsche Forschungsgemeinschaft (DFG) for partial financial support in the initiative FOR 1394, project P3.

\section{References}

[1] Larson R G 1999 The Structure and Rheology of Complex Fluids (New York: Oxford University Press)

[2] Mewis J and Wagner N J 2012 Colloidal Suspension Rheology (Cambridge: Cambridge University Press)

[3] Brader J M 2010 J. Phys.: Condens. Matter 22363101

[4] Hunter G L and Weeks E R 2012 Rep. Prog. Phys. 75066501

[5] Siebenbürger M, Fuchs M, Winter H and Ballauff M 2009 J. Rheol. 53 707-26

[6] Varnik F and Henrich O 2006 Phys. Rev. B 73174209

[7] Besseling R, Weeks E R, Schofield A B and Poon W C K 2007 Phys. Rev. Lett. 99028301

[8] Amann C P et al 2013 arXiv:1302.2030

[9] Voigtmann T 2011 Eur. Phys. J. E 34106

[10] Ikeda A, Berthier L and Sollich P 2012 Phys. Rev. Lett. 109018301

[11] Siebenbürger M, Fuchs M and Ballauff M 2012 Soft Matter 8 4014-24 
[12] Götze W 2009 A Mode-Coupling Theory (Oxford: Oxford University Press)

[13] Brader J M, Cates M and Fuchs M 2008 Phys. Rev. Lett. 101138301

[14] Brader J M, Cates M E and Fuchs M 2012 Phys. Rev. E 86021403

[15] Brader J M, Voigtmann T, Fuchs M, Larson R G and Cates M E 2009 Proc. Natl Acad. Sci. USA 106 15186-91

[16] Henrich O, Weysser F, Cates M E and Fuchs M 2009 Phil. Trans. R. Soc. A 367 5033-50

[17] Krüger M, Weysser F and Fuchs M 2011 Eur. Phys. J. E $341-22$

[18] Amann C P, Siebenbürger M, Krüger M, Weysser F, Ballauff M and Fuchs M 2013 J. Rheol. 57 149-75

[19] Amann C P and Fuchs M 2014 J. Rheol. 58 1191-217

[20] Crassous J J, Siebenbürger M, Ballauff M, Drechsler M, Hajnal D, Henrich O and Fuchs M 2008 J. Chem. Phys. 128204902

[21] Brader J M, Siebenbürger M, Ballauff M, Reinheimer K, Wilhelm M, Frey S J, Weysser F and Fuchs M 2010 Phys. Rev. E 82061401

[22] Siebenbürger M, Ballauff M and Voigtmann T 2012 Phys. Rev. Lett. 108255701

[23] Ballauff M et al 2013 Phys. Rev. Lett. 110215701

[24] Fuchs M and Cates M E 2009 J. Rheol. 53 957-1000

[25] Crassous J J, Siebenbürger M, Ballauff M, Drechsler M, Heinrich O and Fuchs M 2006 J. Chem. Phys. 125204906

[26] Crassous J J, Wittemann A, Siebenbürger M, Schrinner M, Drechsler M and Ballauff M 2008 Colloid Polym. Sci. $286805-12$

[27] Schaertl W and Sillescu H 1994 J. Stat. Phys. 77 1007-25
[28] van Megen W, Mortensen T C, Williams S R and Müller J 1998 Phys. Rev. E 58 6073-85

[29] Lionberger R A and Russel W B 1994 J. Rheol. 38 1885-908

[30] Koumakis N, Laurati M, Egelhaaf S U, Brady J F and Petekidis G 2012 Phys. Rev. Lett. 108098303

[31] Koumakis N, Pamvouxoglou A, Poulos A S and Petekidis G 2012 Soft Matter 8 4271-84

[32] Laurati M et al 2012 J. Phys.: Condens. Matter 24464104

[33] Varnik F, Bocquet L and Barrat J L 2004 J. Chem. Phys. $1202788-801$

[34] Zausch J, Horbach J, Laurati M, Egelhaaf S U, Brader J M, Voigtmann T and Fuchs M 2008 J. Phys.: Condens. Matter 20404210

[35] Zausch J and Horbach J 2009 Europhys. Lett. 8860001

[36] Steif P S, Spaepen F and Hutchinson J W 1982 Acta Metall. 30 447-55

[37] Manning M L, Langer J S and Carlson J M 2007 Phys. Rev. E 76056106

[38] Moorcroft R L, Cates M E and Fielding S M 2011 Phys. Rev. Lett. 106055502

[39] Chen K and Schweizer K S 2010 Phys. Rev. E 82041804

[40] Priya M and Voigtmann T 2014 J. Rheol. 58 1163-87

[41] Voigtmann T, Brader J M, Fuchs M and Cates M E 2012 Soft Matter 8 4244-54

[42] Krüger M, Weysser F and Voigtmann T 2010 Phys. Rev. E 81061506

[43] Fuchs M and Cates M E 2003 Faraday Discuss. 123 267-86

[44] White J L and Metzner A B 1963 J. Appl. Polym. Sci. 71867

[45] Papenkort S and Voigtmann T 2014 J. Chem. Phys 140164507

[46] Chong S-H and Kim B 2009 Phys. Rev. E 79021203

[47] Suzuki K and Hayakawa H 2013 Phys. Rev. E 87012304 